InterSedes, Revista electrónica de las sedes regionales de la Universidad de Costa Rica,

ISSN 2215-2458, Vol XXI, Número 43, Enero - Julio, 2020.

10.15517/isucr.v21i43.41994 | intersedes.ucr.ac.cr | intersedes@ucr.ac.cr

\title{
COMPARACIÓN DE LAS CARACTERÍSTICAS DE PRODUCTIVIDAD ENTRE LAS DISTINTAS VARIEDADES DE UVA DE MAYOR PRODUCCIÓN EN LA VIÑA "EL ESPAVEY" DEL CANTÓN ACOSTA, COSTA RICA
}

\section{PRODUCTIVITY COMPARISON BETWEEN DIFFERENT HIGHEST PRODUCTION GRAPE VARIETIES IN THE "EL ESPAVEY" VINEYARD IN ACOSTA DISTRICT, COSTA RICA}

Michael Fonseca Brenes ${ }^{1}$

Diego Armando Retana Alvarado

María Marta Camacho Álvarez ${ }^{3}$

Iván Badilla Hernández ${ }^{4}$

Recibido: 27.11 .19

Aprobado: 30.04 .20

DOI: $10.15517 /$ isucr.v21i43.41994

\section{Resumen}

El propósito de este artículo es dar a conocer el resultado de la evaluación de la productividad de cinco variedades de uva en el viñedo El Espavey, ubicado en el cantón Acosta (San José, Costa Rica), en sus condiciones respectivas de cultivo (sistemas de siembra en parrilla y espaldera). Se llevó a cabo un muestreo aleatorio de 277 plantas en las zonas denominadas como Alto Espavey y Bajo Espavey. El análisis de los datos se basa en el cálculo de racimos promedio, área foliar máxima media, largo en espaldera para cada muestra por variedad y pruebas de correlación entre la variable número de racimos tanto para con el área foliar máxima estimada como para con la variable de crecimiento. Los resultados sugieren que las variedades Labruscas son las de mayor rendimiento y es en el Alto Espavey y en espaldera donde se desarrolla la mayor producción de la Uva Labrusca que para este estudio se ha denominado Isabella. Este proyecto surgió como una iniciativa de vínculo universidad- sociedad en el marco de la relación existente entre investigación y acción social y de acuerdo con lo estipulado en las políticas universitarias.

Palabras clave: Uvas; Vitis vinifera; Vitis labrusca; sistema de siembra; viñedo; Costa Rica.

\begin{abstract}
This article summarizes the productivity evaluation results of five grape varieties in El Espavey vineyard in Acosta (San José, Costa Rica), considering their growing conditions (i.e. sow on grill and trellis). A random sampling of 277 plants was carried out in the areas called Alto Espavey and Bajo Espavey. Data analysis is based on average clusters, average maximum leaf area, trellis length

${ }^{1}$ Estudiante Universidad de Costa Rica, San Pedro, San José, Costa Rica. Email: michael.fonsecabrenes@ucr.ac.cr

2 Profesor, Universidad de Costa Rica, San Pedro, San José, Costa Rica. Email: diegoarmando.retana@ucr.ac.cr

${ }^{3}$ Profesora, Universidad de Costa Rica, San Pedro, San José, Costa Rica. Email: mariamarta.camacho@ucr.ac.cr

${ }^{4}$ Funcionario Caja Costarricense de Seguro Social, San José, Costa Rica. Email: ibadilllah@ccss.sa.cr
\end{abstract}


of each sample variety (i.e. sow on grill and trellis), and correlation tests between the number of clusters in the estimated maximum leaf area and the growth variable. The results show that the Labrusca varieties have the highest yield and are in Alto Espavey with trellis plantation. This is where the greatest production of the Labrusca grape is developed, and they are named Isabella for the purposes of this study. This project began as a university-society initiative thanks to the relationship between research and social projects stablished on university policies.

Key words: Grapes; Vitis vinifera; Vitis labrusca; planting system; vineyard; Costa Rica.

\section{Introducción}

Entre las políticas de la Universidad de Costa Rica períodos 2016-2020, se menciona el interés de la institución de educación superior por fortalecer el análisis, la discusión y la participación en la solución de los problemas nacionales para plantear propuestas y desarrollar capacidades locales, afianzar la investigación y la acción social, así como el desarrollo artístico, tecnológico y cultural, con el fin de atender los requerimientos de la sociedad costarricense, especialmente de aquellos sectores más vulnerables. Y en las políticas 2021-2025 se señala el interés por generar espacios dialógicos orientados a contribuir con el bienestar nacional e internacional; también menciona propiciar la transferencia del conocimiento generado en las actividades de docencia, investigación y acción social, de manera que contribuya con el desarrollo científico, tecnológico, cultural, social y ambiental del país.

En cumplimiento de las políticas universitarias y en un trabajo conjunto universidad-sociedad, en el año 2019 y 2020 docentes de la Escuela de Formación Docente, estudiantes del proyecto TC 722 ENLACE (entre ellos de Biología) y la Comunidad de Chirraca de Acosta se realizó un proceso de investigación-acción social, relacionado con la producción del vino y los efectos socioeconómicosecológicos.

En términos más biológicos, la vid es un arbusto tipo enredadera perenne perteneciente al género Vitis, nativa de zonas templadas con inviernos no muy rigurosos ya que demanda temperaturas menores a $10{ }^{\circ} \mathrm{C}$ para romper el estado de reposo y requiere de luz y calor para madurar. Esta planta presenta ciclos de producción anual o bianual, posee un ciclo vegetativo y reproductivo realizado de manera conjunta (Loría-Quirós, 2005; Martínez de Toda, 1991; Reynier, 1989). Las uvas son uno de los cultivos de frutas más plantados en el mundo debido en gran medida a la variedad de 
especies existentes y amplia adaptación que permite sean cultivados en todos los continentes y diversos países a nivel mundial (Cahoon, 1985).

Las plantas de uva se reproducen asexualmente a nivel comercial mediante propagación o combinación de estacas a través de injertación, con lo que se logra mantener uniformidad genética, además de alta tasa de multiplicación a costo razonable (Loría-Quirós, 2005). En el caso de la producción de vino, se ha determinado que la calidad varía en función de factores como el clima anual medido en cuatro variables según el ciclo vegetativo: temperatura acumulada, cantidad de lluvia, cantidad de días ventosos y cantidad de días calurosos (Ribéreau-Gayon et al., 1975). Además, de la calidad del suelo en cuanto a nutrientes como N, P, K, Ca, Mg y Fe (Ribéreau-Gayon y Peynaud, 1971).

La mayoría de los viñedos en el mundo se encuentran entre las latitudes $40^{\circ}$ y $50^{\circ} \mathrm{N}$ en el hemisferio norte y entre las latitudes $30^{\circ}$ y $40^{\circ} \mathrm{S}$ en el hemisferio sur, llamado cinturón climático templado (Iland et al., 2009). Por tanto, la viticultura tropical es de origen reciente, con alrededor de sesenta años de antigüedad a nivel comercial (Jogaiah et al., 2013). Las condiciones tropicales propician la ausencia de un periodo de latencia por lo que el crecimiento es ininterrumpido. Con las condiciones de agua y nutrientes adecuada, las plantas se desarrollan en exceso, mostrando un crecimiento continuo de los brotes apicales, aunque puede haber muerte en las yemas de los brotes basales (Camargo, 2005).

El desarrollo de la viticultura en Costa Rica tiende a ser complicado, principalmente por el poco o nulo apoyo que reciben los agricultores interesados en emprender esta práctica y el amplio desconocimiento de las autoridades responsables acerca de este cultivo. Más allá de la información que se puede recopilar de artículos e informes internacionales sobre viticultura tropical, es poco lo conocido sobre las variedades más convenientes o de mejor producción para el tipo de clima en Costa Rica, así como las mejores formas o métodos de cultivo para obtener un mayor rendimiento y rentabilidad al implementarla. Esto provoca que, así como sucede con la viña "El Espavey", se recurra a un sistema de prueba y error con variedades de importancia reconocida en la producción comercial fuera de las fronteras costarricenses pero de los cuales hay pocos registros y valoraciones. 
El viñedo "El Espavey" produce una importante variedad de vinos en un gradiente entre naturalmente dulce y amargo de muy buena calidad, además de la producción de uvas para consumo directo, donde la variedad Isabella (Vitis labrusca) es la de mayor producción y principal fuente de materia prima para este fin. No obstante, se están implementando más de treinta variedades distintas de uvas donde algunas como el Chardonnay, Cabernet Sauvignon o el Merlot son importantes en la producción de vinos a nivel internacional. Esta iniciativa de ampliar la gama de variedades para la producción en el viñedo requiere de un seguimiento en aras de determinar si las nuevas variedades incorporadas son adecuadas y su producción es rentable.

El objetivo principal del estudio que dio origen a este artículo fue evaluar la productividad de las cinco principales variedades actuales del viñedo: Vitis labrusca en adelante llamada Isabella, Isaac (uva que aún no se conoce si es Labrusca o Vitis vinifera), Moldovia (Vitis vinifera), Arcadia (Vitis vinifera) y Labrusca americana (Labrusca), en sus condiciones respectivas de cultivo.

En términos más específicos, se pretendían los siguientes objetivos:

- Comparar el rendimiento de las técnicas de cultivo en espaldera y en parrilla para la misma variedad en las variedades donde se aplican ambas.

- Comparar el rendimiento en la productividad en cuanto a número de racimos de uvas por planta entre las cinco distintas variedades de mayor producción.

- Establecer una posible relación entre variables de crecimiento como ancho a la altura del pecho o extensión longitudinal de la planta con la producción obtenida.

En este sentido, se sostiene que entre mayor crecimiento o desarrollo presenten las plantas, mejores serán los resultados en la producción, además existirá variación inducida por la zona de plantación y técnica de cultivo empleada. Se predice que la variedad Isabella tendrá los mejores resultados por el tiempo de aclimatación y el tamaño de las plantas en función al tiempo que llevan en el viñedo. 


\section{Marco teórico}

La vid es una planta cuyas raíces son de crecimiento rápido para cumplir funciones de anclaje, absorción de agua y minerales, además de acumular reservas energéticas como almidón (AlmanzaMerchán, 2011). En este proceso, la raíz tiene un periodo de entre 7 a 10 años para colonizar el suelo y posteriormente por un periodo de entre 10 a 40 años de explotación del suelo previo a decaer alrededor de los 50 años (Martínez de Toda, 1991). En cuanto al tallo o tronco, este recibe el nombre de parra, pie o cepa donde la longitud depende de la poda de formación según el sistema de producción que se emplee. Su color es castaño (más oscuro es algunas variedades), y su corteza es más rigurosa según la edad de la planta y son los brazos los encargados de la conducción de nutrientes hacia la vegetación y los frutos, así como delimitar el espacio ocupado (GrimaltTrò, 2016).

Por otra parte, Costa Rica se encuentra en una zona ubicada entre el trópico de Cáncer y el de Capricornio (entre los $23^{\circ}$ Norte y Sur), esto se torna importante al considerar la variación climática que representa el trópico para la especie (o variedad) de la uva, ya que estas regiones son más cálidas al estar más cerca del sol, donde incluso éste alcanza un punto directamente sobre la cabeza al menos una vez por año a diferencia de las zonas templadas. Otro factor es la inclinación axial del planeta, la cual afecta con mayor fuerza a esta zona provocando mayor variación en el eje del sol en relación con la Tierra, creando variación entre el lugar donde sale y se oculta el sol al año (Demir, 2014), esto afecta no solo el cultivo de la uva, sino también la calidad esperable (Commins et al., 2012).

En este sentido, se ha observado que los cultivares de uva presentan comportamientos diversos en diferentes lugares del trópico debido a la variación climática, como resultado de la altitud (creando microclimas), o por la duración de las estaciones seca o lluviosa y la nubosidad (Camargo, Pereira y Guerra, 2010). Es imperativo realizar una evaluación de las condiciones climáticas de una determinada zona antes de establecer este tipo de cultivo y así seleccionar el cultivar más adecuado, además de evaluar el tipo de producto que se podría favorecer en dicho lugar.

En zonas de baja altitud donde no hay una gran variación de temperatura entre día y noche resulta en uvas con niveles altos de azúcar, baja acidez, alto $\mathrm{pH}$ y baja cantidad de taninos, antocianinas y 
polifenoles. Sin embargo, normalmente se prefieren zonas de mayor altitud para el cultivo de uva al tener temperaturas más suaves que promueven un lento desarrollo de la vegetación y un período más largo de uvas maduras. Se sabe que la ocurrencia de noches más frías favorece la acumulación de componentes de calidad de uvas rojas como antocianinas y taninos y en las uvas blancas se mejora la intensidad y calidad del aroma (Camargo, Pereira y Guerra, 2010). No obstante, la radiación solar más alta incidente del altiplano tropical es el factor más importante para garantizar una excelente calidad del vino y la planta se adapta a una importante cantidad de tipos de suelos donde destacan los franco-arenosos y silicio-calizos como promotores de una alta calidad en el vino (Almanza-Merchán, 2011).

Otros factores para el proceso de vinificación son la composición fisicoquímica, conformación y número del microbiota en la periferia de la baya madura (Tuanama y Smith, 2019). En el caso de las uvas tintas se toman muy en cuenta factores como el peso de la baya y la densidad del mosto o zumo y los factores como la relación de azúcares y acidez son buenos indicadores en la determinación del momento idóneo para el tiempo de vendimia y calidad de la uva (Coombe, 1987; González y Díez, 1992). Los compuestos fenólicos de las bayas son de igual importancia siendo factores importantes en el color, sabor y cuerpo de los vinos tintos (Pinelo et al., 2006).

Según, Camargo (2005), para realizar una viticultura exitosa es necesario proporcionar condiciones adecuadas a la planta desde que es un vástago. En general, la necesidad de constante riego en periodos secos es una norma, así como una cantidad abundante de abono orgánico, además del cuido del potencial efecto de los elementos químicos del suelo. Durante la formación de la planta aparece una forma conocida como dosel (en el primer ciclo vegetativo), donde debe ser muy fuerte para resistir un periodo de emisión abundante y crecimiento de brotes axilares. Dado que los brotes laterales son proporcionales al brote principal, es necesario eliminar los excedentes podándolos para obtener la densidad adecuada.

Para analizar el rendimiento de una cosecha de uvas en condiciones de siembra en clima tropical se deben tomar en cuenta ciertas características particulares, tales como la presencia de dos cosechas anuales. En la mayoría de los países con dichas condiciones, incluyendo países latinoamericanos como Guatemala, Brasil o Colombia, lo que genera un rendimiento 
significativamente más alto que en otros países (templados), incluso en algunas cosechas se aplica un ciclo continuo de cinco meses (Possingham, 2006). En estos países, las vides son de hoja perenne pero las características que lo permiten aún no son claras; sin embargo, con base en la observación se sugiere que existen condiciones mínimas como temperatura promedio superior a los $15^{\circ} \mathrm{C}$, lo que evitaría la senescencia y caída de hojas, pero es posible que interactúen con otros factores como estrés hídrico y carga de cultivos (Possingham, 2006).

A pesar de que a nivel mundial existen unos 8000 cultivares de uva, solo una fracción de estas son utilizadas para fines comerciales, y pueden clasificarse según su color como: blancas, negras, rojas o rosadas, además del uso que se le da para: mesa, vinos o pasas (Pardo, 2016). La principal uva utilizada en el viñedo "El Espavey" es, como se mencionó anteriormente, la variedad Isabella (Vitis $s p$.), uva de color negro utilizada comúnmente para la elaboración de jugos, pulpas, mermeladas y vinos, y que además es de origen americano altamente resistente a enfermedades que afectan con mayor frecuencia a otros cultivares, facilitando su uso en condiciones de trópico donde se favorece la proliferación de hongos durante prácticamente todo el año (FAO, 2010).

\section{Diseño metodológico}

En el II Semestre de 2019 se llevó a cabo un estudio en el viñedo "El Espavey" ubicado en la zona

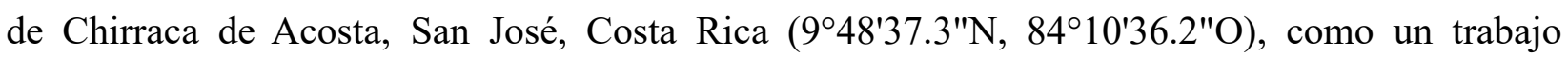
conjunto de docentes y estudiantes de la Universidad de Costa Rica y los responsables de la viña. El Espavey surge a principios de 2004 como un proyecto agroturístico con fines recreativos. Hasta ese momento, la finca en que se ubica el viñedo estuvo dedicada al cultivo de cítricos y café. A finales de 2013, se vislumbra su evolución hacia el desarrollo de una empresa con implicaciones organizativas, productivas y de mercadeo, en el marco de tipologías emergentes en Costa Rica como el enoturismo o turismo enológico focalizado en zonas de producción vinícolas. También, se destaca el vititurismo que implica actividades dentro de la plantación de la uva, así como la producción del vino en la bodega.

La literatura científica evidencia la relevancia que este ámbito ha adquirido, particularmente en países europeos como España con gran tradición en la producción de vino, pues las motivaciones e intereses de los turistas han cambiado hacia destinos que aporten nuevas experiencias. En este 
sentido, la demanda de rutas del vino adaptadas al perfil del enoturista representa una potente línea de investigación (López-Guzmán, Rodríguez García, y Vieira Rodríguez, 2013).

Actualmente, El Espavey se encuentra en una etapa de experimentación, cuenta con alrededor de 35 variedades de uva como Cabernet Sauvignon, Garnacha, Tempranillo, Zinfandel y Torontel, procedentes de distintas regiones del mundo, las cuales siguen un proceso de tropicalización.

Para el diseño de la metodología experimental, se tomaron datos en dos zonas particulares dentro de la misma finca a las que se denominó Alto Espavey y Bajo Espavey según la altura en que se ubicaban (los nombres se asocian a una diferencia en altura y exposición al sol). Allí se extrajeron cinco tipos de uvas de las variedades: Isabella, Isaac, Moldovia, Arcadia y Labrusca americana.

De cara al análisis, Isaac se operacionaliza como una morfoespecie, es decir, como una especie con características distintas a las demás pero que no ha sido identificada. Cabe señalar que la uva Isaac, recibe ese nombre dado que el Sr. Iván Badilla Hernández la encontró en el jardín de una casa en la provincia de Cartago, Costa Rica. Se desconoce el nombre científico, pero resultó ser un buen ejemplar por su capacidad de adaptación al trópico. Entonces, le dio el nombre de su hijo, mientras se determina su taxonomía mediante los estudios correspondientes.

La Isabella (Vitis labrusca) es un híbrido natural de la familia Vitaceae procedente del Sur de California, conocida como Chinche, Monterrico o Frambua en contextos latinoamericanos. La variedad Isabella es una variedad diferente a la Labrusca americana por el lugar y forma de domesticación pese a ser la misma especie. Dado que Isabella es la de mayor abundancia, se tomaron dos muestras de 60 individuos al azar de las zonas denominadas como Bajo Espavey en los sistemas de siembra en parrilla y espaldera respectivamente (existe una variación en la altura entre ambas formas de cultivo), y una tercera muestra de la zona denominada como Alto Espavey en espaldera.

Para las variedades Isaac, Moldovia, Arcadia se tomó una única muestra de plantas en espaldera de 41 individuos, 53 individuos y 60 individuos respectivamente. Finalmente, se tomaron dos 
muestras de labrusca americana de 50 y 13 individuos respectivamente, donde la primera muestra pertenece a la forma de cultivo en espaldera y la segunda a parrilla.

Cabe mencionar que la variación en las muestras se debieron principalmente a disponibilidad del material vegetal, tanto para aquellos que estaban sembrados solo bajo un sistema de siembra (espaldera o parrilla), como para aquellas en las que no había suficientes individuos para completar la muestra fijada de 30 individuos. Sin embargo, se despreciaron de este análisis, las variedades que apenas están siendo introducidas y de las que se cuenta con muy poco material, con el fin de obtener un promedio adecuado.

Una vez seleccionada cada planta al azar (a excepción de aquellas donde la muestra era apenas la necesaria), se procedió a seleccionar 3 hojas por individuo para obtener los valores de largo y ancho (cm) necesario para calcular un área foliar máxima estimada y obtener el promedio por planta. Además, con el fin de estimar la producción por planta, se contabilizaron los racimos de uvas por individuo. Finalmente, como variables de crecimiento, para aquellas variedades plantadas en parrilla se obtuvo el ancho del tallo a la altura del pecho $(\mathrm{cm})$ y para las variedades en espaldera (cuya altura se mantiene intencionalmente baja), se obtuvo el valor de la extensión (cm) a lo largo de la espaldera.

Para el análisis de datos se calcularon los valores de racimos promedio, área foliar máxima promedio y largo en espaldera (o ancho a la altura del pecho), para cada muestra por variedad.

Además, se obtuvo un valor corregido de número promedio de frutos eliminando la ausencia para comparar con los resultados obtenidos, esto teniendo en cuenta que no todas las plantas están en las mismas etapas de desarrollo y especialmente en variedades menos comunes. Posteriormente, se realizaron pruebas de correlación entre el número de racimos tanto para con el área foliar máxima estimada como para con la variable de crecimiento (según el caso). En las variedades de Isabella y Labrusca americana se compararon los resultados con la misma variedad en condiciones distintas de altura y siembra. Todas las mediciones se realizaron con una cinta métrica de $5 \mathrm{~m}$ de longitud.

En el siguiente apartado se presentan los resultados que permiten verificar el objetivo principal del estudio que antecede a este artículo, que es evaluar la productividad de las cinco principales variedades de la Finca El Espavey en sus condiciones respectivas de cultivo. 


\section{Resultados}

En cuanto a producción medida en número de racimos, los mejores resultados se obtuvieron en las variedades Isabella, en condiciones de espaldera (en Alto Espavey) y en parrilla, así como en Labrusca americana con cantidades promedio superiores a los 17 racimos. En contraparte, los resultados menos favorables fueron para las variedades Moldovia y Arcadia con 0.13 y 0.93 individuos en promedio respectivamente, esto se mantiene incluso al eliminar las plantas con cero producciones del promedio (ver Tabla 1 más adelante).

\section{Tabla 1}

Promedio y desviación estándar de la cantidad de racimos, área foliar máxima y extensión en espaldera o ancho a la altura del pecho para las distintas variedades de uvas.

\begin{tabular}{|c|c|c|c|c|c|c|}
\hline Variedades & $\begin{array}{l}\text { Forma de } \\
\text { cultivo }\end{array}$ & $\begin{array}{l}\text { Número de } \\
\text { racimos } \\
\text { promedio }\end{array}$ & $\begin{array}{l}\text { Número de } \\
\text { racimos } \\
\text { promedio sin } \\
\text { ausencia }\end{array}$ & $\begin{array}{l}\text { Área foliar } \mathrm{E} \\
\text { máxima es } \\
\left(\mathrm{cm}^{2}\right)\end{array}$ & $\begin{array}{l}\text { Extensión } \\
\text { es espaldera } \\
\text { (cm) }\end{array}$ & $\begin{array}{l}\text { Ancho a } \\
\text { la altura } \\
\text { del pecho } \\
(\mathrm{cm})\end{array}$ \\
\hline Isabella-bajo & Espaldera & $5.23( \pm 11.33)$ & $8.97( \pm 13.72)$ & $\begin{array}{c}270.96 \\
( \pm 106.96)\end{array}$ & $\begin{array}{c}173.33 \\
( \pm 80.06)\end{array}$ & NA \\
\hline Isabella-alto & Espaldera & $21.78( \pm 13.55)$ & $22.53( \pm 13.15)$ & $334.34( \pm 68.88)$ & $\begin{array}{c}184.93 \\
( \pm 47.44)\end{array}$ & NA \\
\hline Isabella & Parrilla & $17.17( \pm 29.96)$ & $42.92( \pm 33.89)$ & $233.04( \pm 85.42)$ & NA & $\begin{array}{c}11.17 \\
( \pm 9.09)\end{array}$ \\
\hline Moldovia & Espaldera & $0.13( \pm 0.59)$ & $1.75( \pm 1.50)$ & $\begin{array}{c}169.83( \pm \\
68.44)\end{array}$ & $\begin{array}{c}115.76 \\
( \pm 75.91)\end{array}$ & NA \\
\hline Arcadia & Espaldera & $0.93( \pm 2.13)$ & $3.73( \pm 2.92)$ & $167.25( \pm 76.16)$ & 5) $\begin{array}{c}99.40 \\
( \pm 63.06)\end{array}$ & NA \\
\hline Isaac & Espaldera & $2.24( \pm 4.19)$ & $4.84( \pm 5.07)$ & $\begin{array}{c}159.07 \\
( \pm 102.96)\end{array}$ & $\begin{array}{c}105.54 \\
( \pm 94.82)\end{array}$ & NA \\
\hline $\begin{array}{l}\text { Labrusca } \\
\text { americana }\end{array}$ & Espaldera & $5.58( \pm 8.65)$ & $10.33( \pm 9.49)$ & $217.92( \pm 68.84)$ & 4) $\begin{array}{c}196.91 \\
( \pm 73.14)\end{array}$ & NA \\
\hline $\begin{array}{l}\text { Labrusca } \\
\text { americana }\end{array}$ & Parrilla & $27.50(34.90)$ & $47.14( \pm 33.94)$ & $250.30( \pm 51.52)$ & NA & $\begin{array}{c}8.03 \\
( \pm 5.70)\end{array}$ \\
\hline
\end{tabular}

Nota: $\mathrm{NA}=$ no aplica. Fuente: elaboración propia.

Al observar la variable de área foliar máxima se puede constatar que las variedades de mayor área son aquellas de mayor producción, siendo Isabella del Alto Espavey la de mayores tamaños con $334.34 \mathrm{~m} 2$ ( $(68.88 \mathrm{~m} 2)$ en promedio. Las variedades Isaac, Arcadia y Moldovia son las que presentan las menores aéreas foliares máximas con $159.07 \mathrm{~m} 2( \pm 102.96 \mathrm{~m} 2), 167.25( \pm 76.16 \mathrm{~m} 2)$ y $169.83 \mathrm{~m} 2( \pm 68.44 \mathrm{~m} 2)$ respectivamente. 
Por otra parte, para variables en espaldera, las de mayores extensiones fueron nuevamente Labrusca americana e Isabella (en Alto Espavey) con $196.91 \mathrm{~m}( \pm 73.14 \mathrm{~m})$ y $184.93 \mathrm{~m}( \pm 47.44 \mathrm{~m})$ respectivamente (ver Tabla 1$)$.

Al correlacionar las variables en función al número de racimos, se observó que no parece existir mayor correlación entre el número de racimos con el área foliar máxima, donde la única variedad con un resultado importante fue Isaac $(r=0.65)$ y algunos indicios en Isabella en espaldera del Alto Espavey y en Moldovia (ver Tabla 2 y Figura 1 más adelante).

Tabla 2

Correlaciones entre el área foliar máxima y el número de frutos.

\begin{tabular}{ccc}
\hline Variedad & Forma de cultivo & $\begin{array}{c}\text { Correlación Área foliar } \\
\text { máxima- número promedio } \\
\text { de racimos (r) }\end{array}$ \\
\hline Isabella-bajo & Espaldera & 0.15 \\
Isabella-alto & Espaldera & 0.33 \\
Isabella & Parrilla & 0.01 \\
Moldovia & Espaldera & 0.03 \\
Arcadia & Espaldera & 0.32 \\
Labrusca & Espaldera & 0.07 \\
Labrusca & Parrilla & 0.33 \\
Isaac & Espaldera & 0.65 \\
\hline
\end{tabular}

Fuente: elaboración propia.

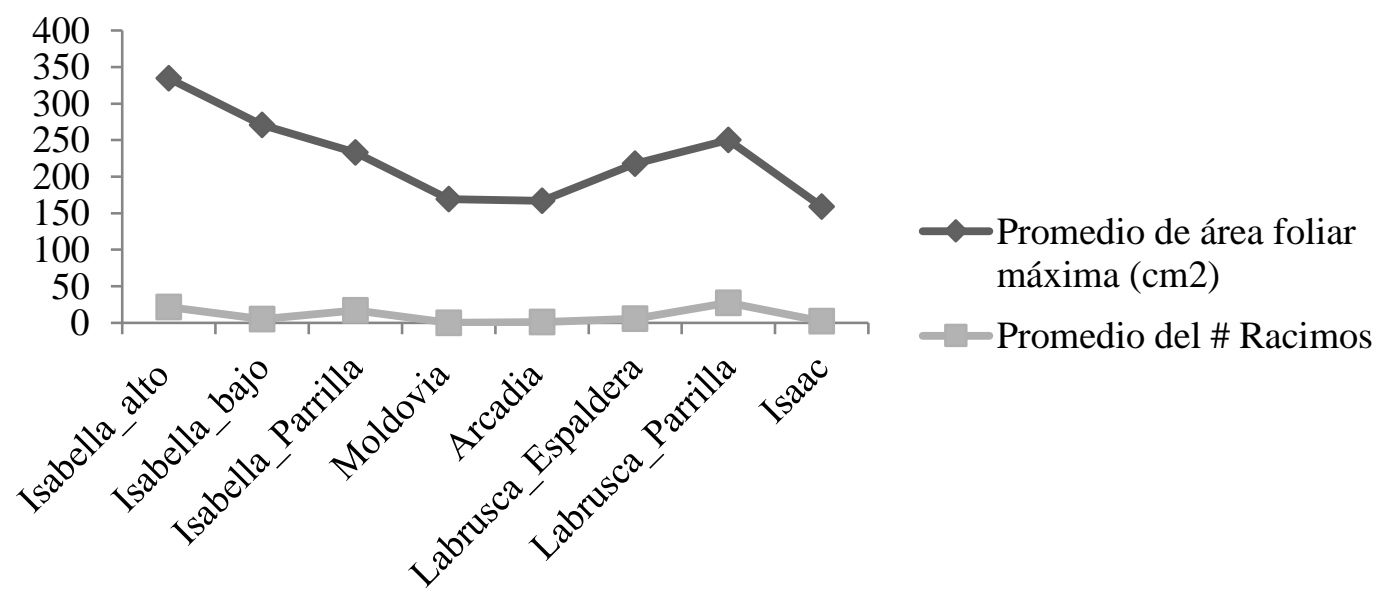

Figura 1. Relación entre el área foliar máxima y el número de frutos en todas las variedades. Fuente: elaboración propia. 
En cuanto a las variables de distancia en espaldera y ancho a la altura del pecho, parecen tener un mayor efecto sobre la productividad en término de racimos, ya que las correlaciones son mayores, principalmente en las variedades en espaldera de Isabella bajo Espavey ( $\mathrm{r}=0.53)$, Arcadia $(\mathrm{r}=0.46)$ e Isaac $(r=0.50)$ y las variedades en parrilla de Isabella $(r=0.64)$ y Labrusca $(r=0.65)$ (Tabla 3; Figura 2 más adelante).

Tabla 3

Correlaciones entre las variables de extensión de espaldera y ancho a la altura del pecho con respecto a área foliar y promedio de frutos.

\begin{tabular}{cccccc}
\hline Variedad & $\begin{array}{c}\text { Forma de } \\
\text { cultivo }\end{array}$ & $\begin{array}{c}\text { Extensión en } \\
\text { espaldera } \\
\text { Área foliar }\end{array}$ & $\begin{array}{c}\text { Extensión en } \\
\text { espaldera - } \\
\text { Promedio de } \\
\text { Frutos }\end{array}$ & $\begin{array}{c}\text { Ancho a la } \\
\text { atura del } \\
\text { pecho - Área } \\
\text { foliar }\end{array}$ & $\begin{array}{c}\text { Ancho a la } \\
\text { atura del } \\
\text { pecho - } \\
\text { Promedio de } \\
\text { Frutos }\end{array}$ \\
\hline Isabella_bajo & Espaldera & 0.47 & 0.53 & NA & NA \\
Isabella_alto & Espaldera & 0.16 & 0.24 & NA & NA \\
Isabella & Parrilla & NA & NA & 0.08 & 0.64 \\
Moldovia & Espaldera & 0.74 & 0.22 & NA & NA \\
Arcadia & Espaldera & 0.61 & 0.46 & NA & NA \\
Labrusca & Espaldera & 0.09 & 0.25 & NA & NA \\
Labrusca & Parrilla & NA & NA & 0.30 & 0.65 \\
Isaac & Espaldera & 0.83 & 0.50 & NA & NA \\
\hline
\end{tabular}

Nota: $\mathrm{NA}=$ no aplica. Fuente: elaboración propia.

En lo que respecta al área foliar máxima en función a la forma de cultivo, también se obtienen correlaciones importantes en espaldera para Isabella en el bajo Espavey ( $\mathrm{r}=0.47)$, Moldovia $(\mathrm{r}=0.74)$, Arcadia $(\mathrm{r}=0.61)$ e Isaac $(\mathrm{r}=0.83)$, aunque no hay correlaciones relevantes con ancho a la altura del pecho (Tabla 3; Figura 3 más adelante). 


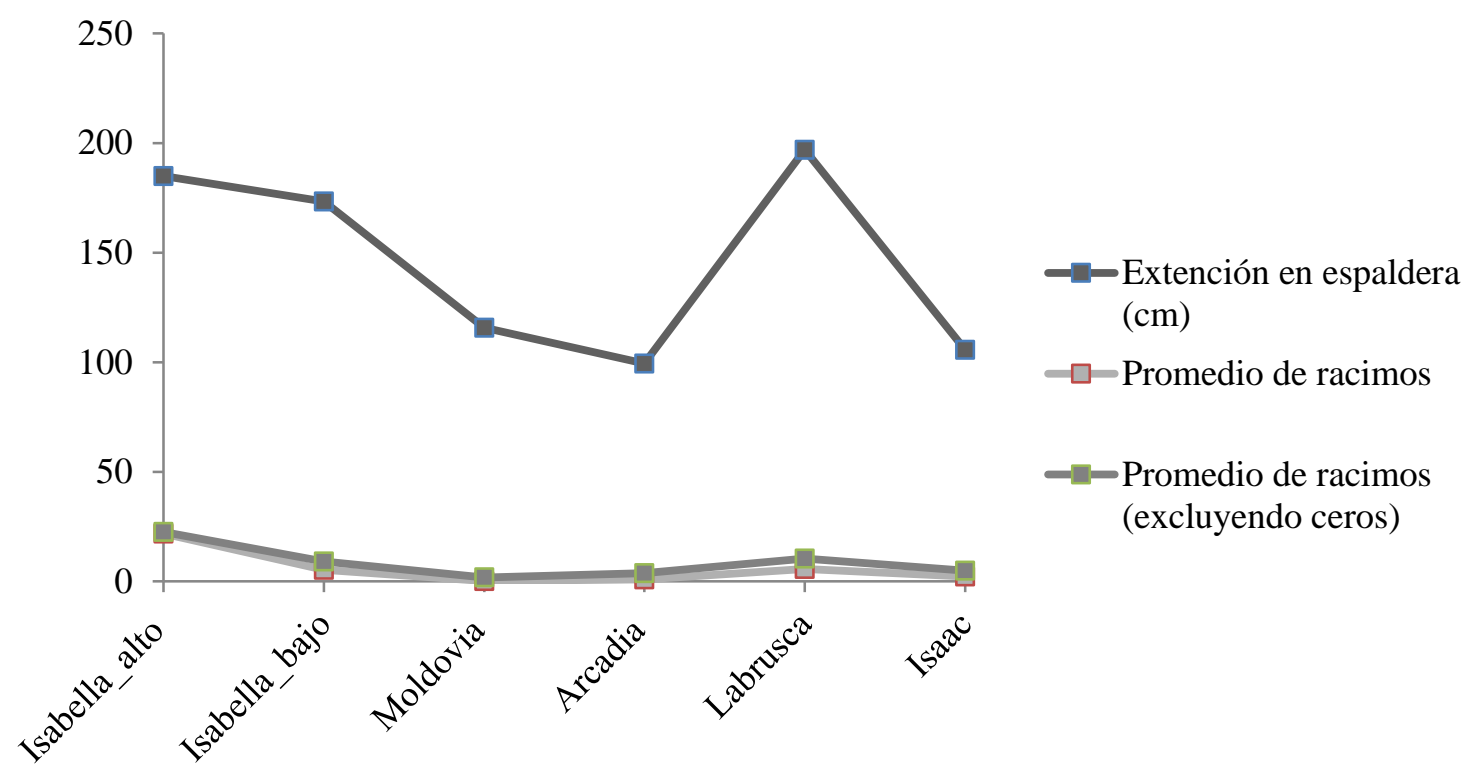

Figura 2. Relación entre el número de racimos producidos con respecto a la extensión de la planta en la espaldera. Fuente: elaboración propia.

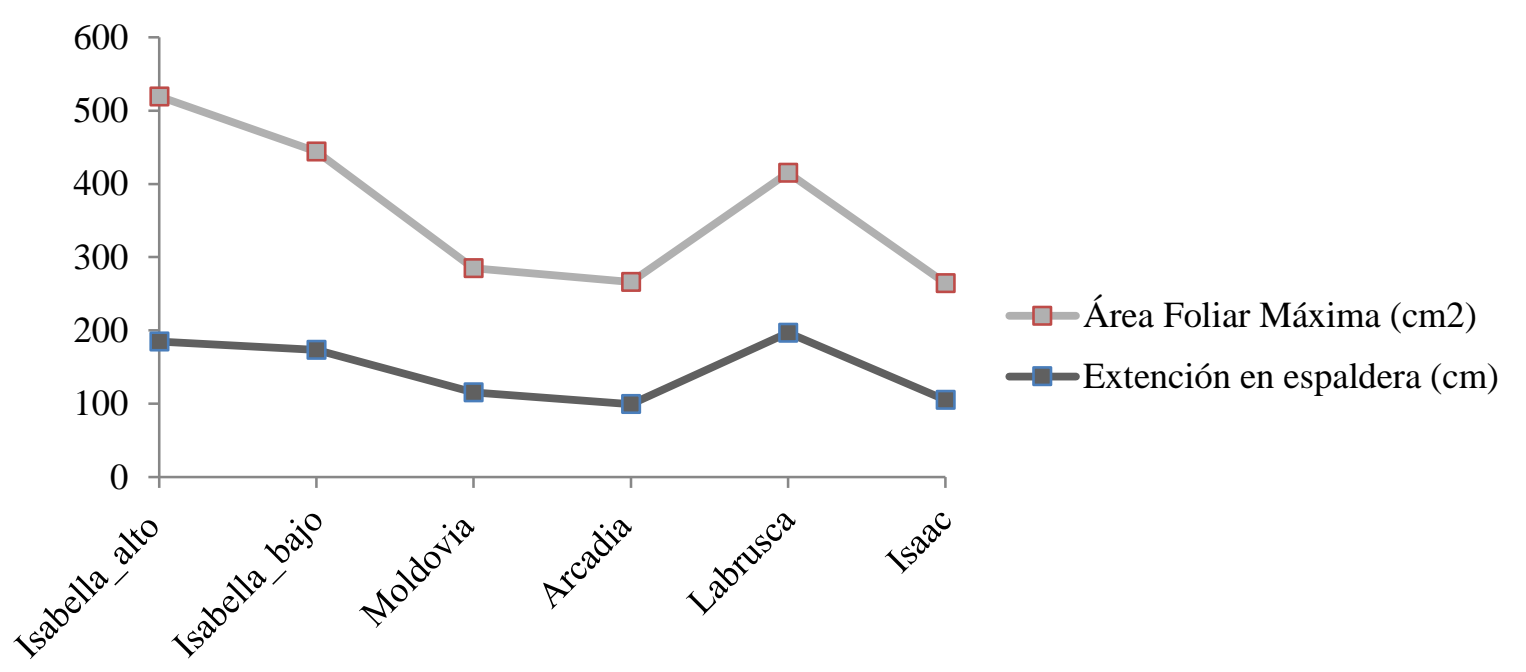

Figura 3. Relación entre el área foliar máxima con respecto a la extensión de la planta en la espaldera. Fuente: elaboración propia. 
Finalmente, se correlacionaron las variables de las variedades en espaldera (Labrusca americana e Isabella) mostrando muchas similitudes, incluyendo el número de racimos (Figura 4; Figura 5). Además, se comparó el rendimiento de Isabella en las tres formas empleadas en este análisis (espaldera alto y bajo, y parrilla), donde Isabella en alto espaldera fue la que tuvo los mejores resultados en cuanto a número de racimos y área foliar máxima (Figura 6 más adelante). Al hacer lo mismo con Labrusca americana se obtuvo un mejor rendimiento al sembrarla en parrilla (Figura 7 más adelante).

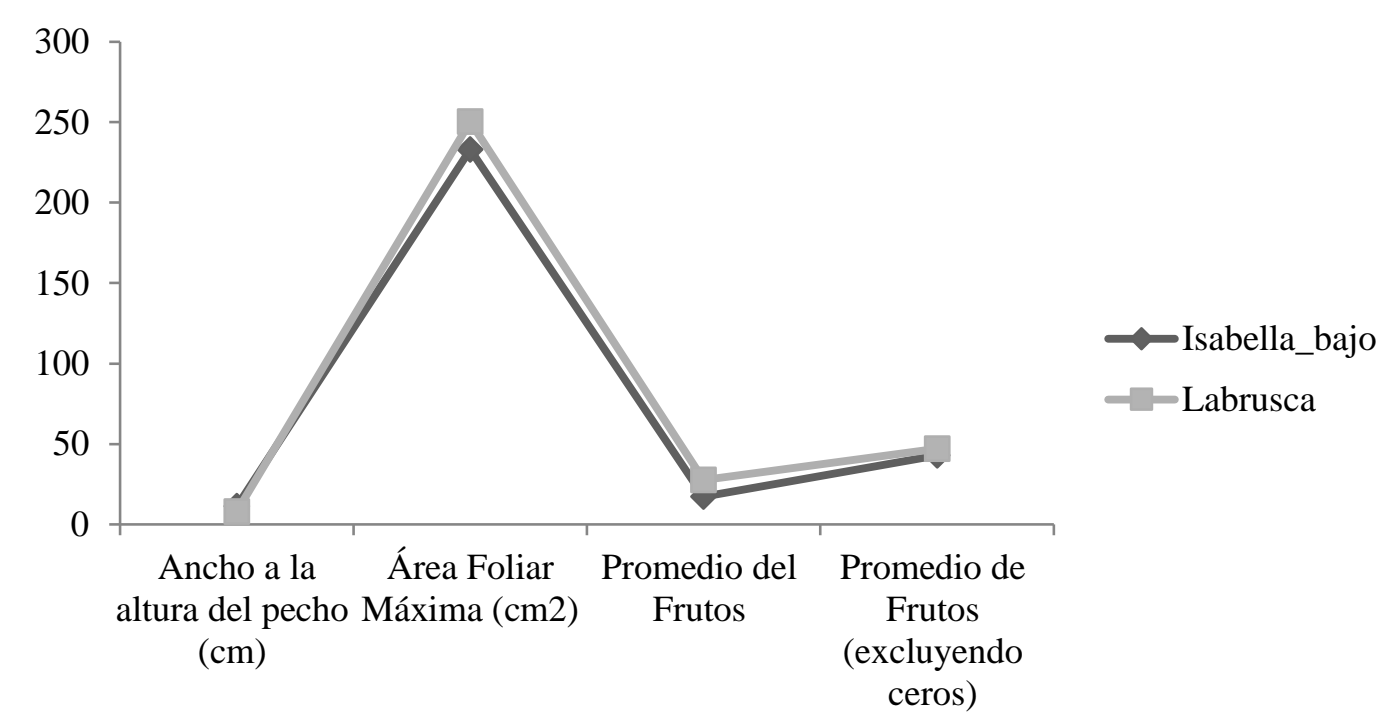

Figura 4. Relación entre Isabella y Labrusca americana con respecto a ancho a la altura del pecho contra área foliar y promedio de racimos. Fuente: elaboración propia.

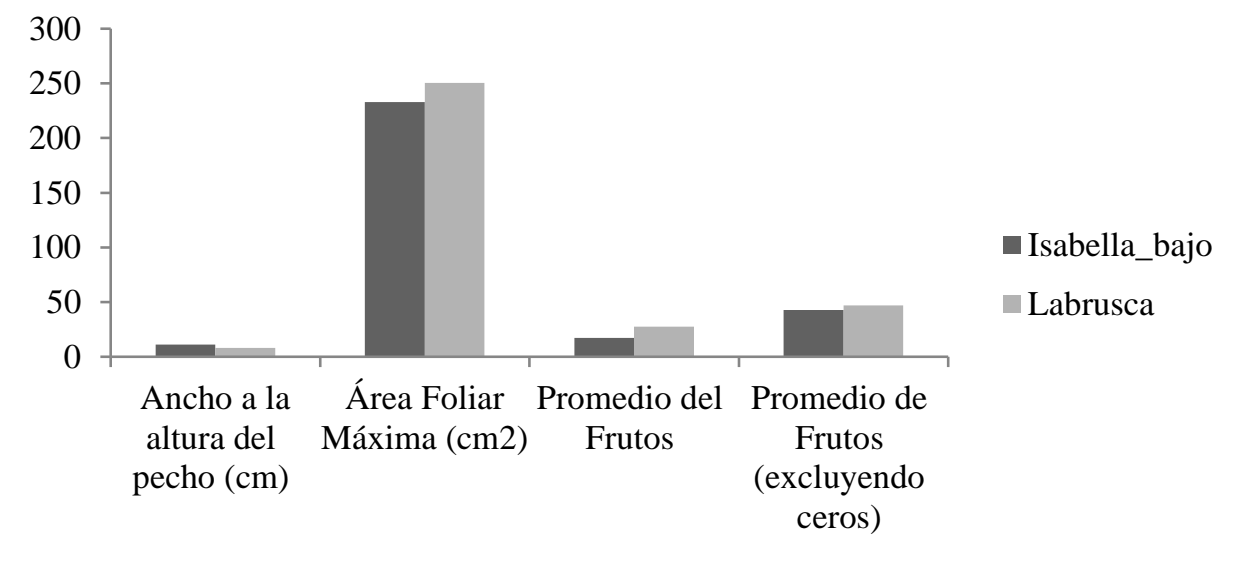

Figura 5. Comparación entre Isabella y Labrusca americana en cuanto a ancho a la altura del pecho, área foliar y promedio de racimos. Fuente: elaboración propia. 


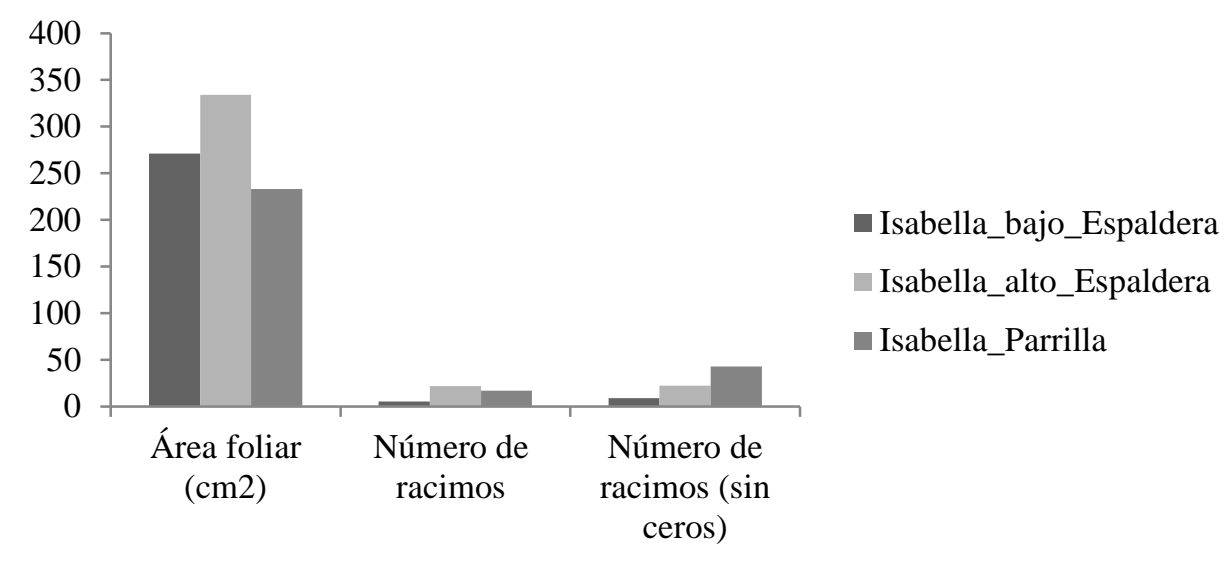

Figura 6. Comparación del rendimiento en Isabella según forma de cultivo (espaldera o parrilla) en cuanto a área foliar y número de racimos. Fuente: elaboración propia.

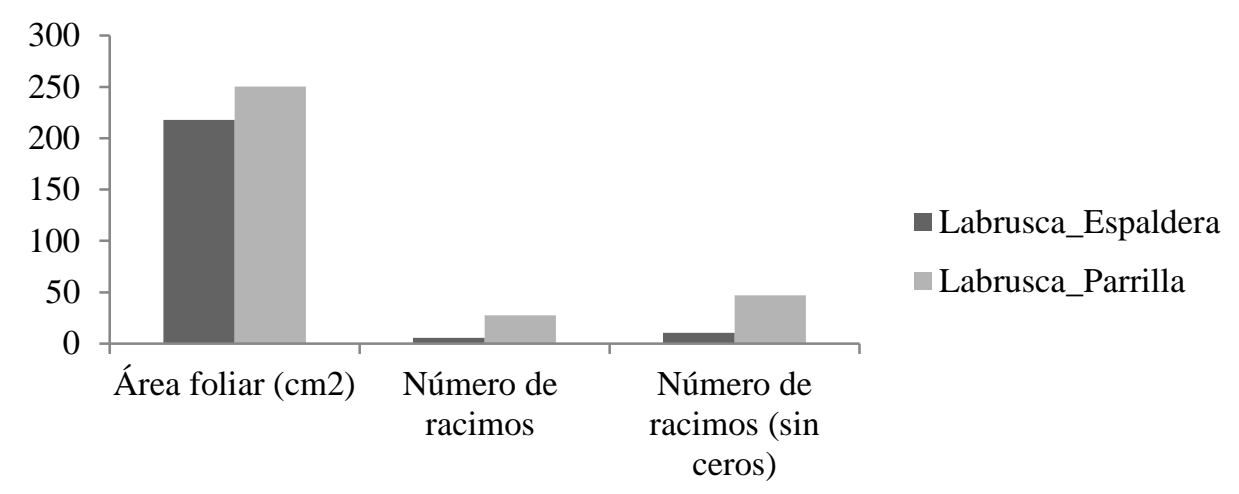

Figura 7. Comparación del rendimiento en Labrusca americana según forma de cultivo (espaldera o parrilla) en cuanto a área foliar y número de racimos. Fuente: elaboración propia.

Estos datos surgen de la comparación del rendimiento de las técnicas de cultivo en espaldera y en parrilla para la misma variedad en las variedades donde se aplican ambas, del rendimiento en la productividad en cuanto a número de racimos de uvas por planta entre las cinco distintas variedades de mayor producción y la relación entre variables de crecimiento como ancho a la altura del pecho o extensión longitudinal de la planta con la producción obtenida. Temas que se discutirán en el siguiente apartado.

\section{Discusión}

Para ser competitivos en el mercado de la viticultura se requiere de una eficacia importante en la producción, así como una calidad que respalde el producto (Bonilla, 2015). Además, de factores como la situación edáfica y climatológica en el viñedo, otros como la genética (en cuanto a 
variedad, clon o injerto), y el manejo humano que se le suministre (como las técnicas de cultivo elegidas) son de vital importancia para mantener una vegetación sana con vigor y una exposición adecuada de los racimos para evitar daño (Bonilla, 2015). Es por estas razones, que realizar una comparación entre variables de crecimiento de distintas variedades o entre técnicas de cultivo para la misma variedad pueden contribuir a dilucidar cuáles son las de mayor producción.

Como se sabe, la principal función de las hojas en plantas es realizar la fotosíntesis de manera eficiente, por lo que se requiere mantener plantaciones con follajes de óptimo vigor, además de una distribución relativamente uniforme para obtener fotosintatos suficientes que faciliten la producción de frutos de amplia calidad (Almanza, 2005). En consecuencia, la poda y un buen manejo cobra importancia no solo en prevención de plagas y enfermedades, sino también en calidad del producto (Almanza, 2005). El área foliar está fuertemente relacionada con el nivel que se intercepta la luz, además de la transpiración y fotosíntesis neta de la copa, aunque varía según las condiciones ambientales (Battaglia et al., 1998).

Pese a que los datos obtenidos en el viñedo muestran que no parece existir una correlación importante entre área foliar máxima con la producción de racimos, sí hay leves correlaciones para todas las variedades, además se observa que los mejores resultados en producción los tienen las variedades más comunes y con mayor tiempo de aclimatación (como Isabella y Labrusca americana), lo que parece concordar mejor con la literatura. En varias referencias se puede encontrar que la relación entre área foliar contra carga óptima de frutos por planta ha sido sujeto de estudio de fisiólogos a nivel mundial y pese a que los resultados varían según la región y tipo de clima, se han reportado números como $1 \mathrm{~m}^{2}$ por kilogramo en climas cálidos y soleados y 1.5 $\mathrm{m}^{2}$ por kilogramo en condiciones más frescas (Disegna, Coniberti y Dellacassa, 2005). No obstante, un gran número de racimos puede generar una influencia desfavorable de una enorme producción sobre la calidad de los vinos que se atribuye a un desbalance en los centros productores de azúcar durante la fotosíntesis (Disegna et al., 2005).

Las otras variables de crecimiento que son extensión en espaldera $(\mathrm{cm})$ y ancho a la altura del pecho $(\mathrm{cm})$, parecen estar más relacionadas con el número de racimos en los datos recopilados. Ambas variables, aunque distintas, muestran el desarrollo de la planta, aumentando en el primer 
caso el área efectiva y en el segundo caso midiendo el estado de desarrollo, permitiendo inferir entre otras cosas un área de cobertura vegetal mayor. Diversos estudios han demostrado que la concentración de azúcares en la baya está estrechamente relacionada con el equilibrio entre el crecimiento vegetativo y la producción de la cepa (Keller et al., 2004; Kliewer y Weaver, 1971).

No obstante, debe haber un equilibrio en número de hojas con el número de frutos para no afectar la producción (estimado entre 7 y $14 \mathrm{~cm}^{2}$ de hoja por cada gramo de uva) (Uriarte et al., 2014). La anchura del tallo o largo en espaldera pueden ser formas de medir esta variable indirectamente, pero tomando en cuenta que el equilibrio óptimo está sujeto a variables (condiciones ambientales, variedad y cantidad de luz interceptada por unidad de área foliar, entre otros), solo experimentalmente se puede determinar que es conveniente permitir el crecimiento en espaldera de forma productiva, así como el follaje en plantas de gran envergadura.

La figura 6 permite observar un aparente mayor beneficio de sembrar en espaldera que en parrilla para Isabella, en cuanto a número de racimos y área foliar máxima. Se debe tomar en cuenta que la técnica de cultivo en espaldera tiene ciertas ventajas a nivel agronómico: posible cosecha nocturna para evitar la oxidación y mantener el aroma, posibilidad de injertar más plantas en el mismo terreno (más de 500 cepas por hectárea) y facilidad en la movilidad entre parcelas o calles optimizando la cosecha. Además, de una mejor eficiencia de los tratamientos sanitarios por lo que se reducen los riesgos de padecer enfermedades relacionadas con el exceso de humedad como el mildiú. Finalmente, puede haber una mejor maduración de la uva al recibir mayor cantidad de rayos solares, entre otros (Sánchez et al., 1999). Si estas ventajas fueron concebidas en comparación a la siembra en vaso, algunas se pueden extrapolar a la parrilla como la facilidad de manipulación, cosecha y cuidados.

Como se mencionó anteriormente, Isabella es la variedad de mayor producción en el viñedo El Espavey (al sumar el total de la producción). Además, los datos sugieren que es en el alto y en espaldera donde se da la mejor producción. En este caso, la variación de altura no es tan drástica como para representar un problema, pues se encuentra en el cantón de Acosta cuya altura máxima no supera los 1200 m.s.n.m. y la altura promedio en Chirraca ronda los 1000 m.s.n.m. (Herrero et al., 1993), además es la zona donde se tiene una mejor exposición al sol y como se mencionó anteriormente, esta planta requiere dosis importantes de luz solar para alcanzar su mayor 
efectividad, por lo que es probablemente la mejor zona para producción. A pesar de los resultados de parrilla, fueron muy similares para las dos variedades en que se midieron (Isabella y Labrusca americana) alcanzando valores bastante altos, incluso en Labrusca se observó mayor producción en espaldera.

\section{Conclusiones y recomendaciones}

Los resultados permiten concluir que el mayor rendimiento está en Isabella y en Labrusca americana, incluso Labrusca supera a Isabella en parrilla (además de obtener muy buenos resultados en espaldera). No obstante, si se toma en cuenta el crecimiento que se observó en variedades como Moldovia, Arcadia o Isaac, donde muchas plantas apenas estaban creciendo y por la baja densidad de muestra se contabilizaron plántulas muy pequeñas generando varios ceros en la muestra, es posible afirmar que los resultados obtenidos en este análisis cambien con el tiempo. En estas circunstancias, las dos primeras variedades mencionadas son las de mejor rendimiento.

Por otra parte, la comparación entre espaldera y parrilla en Labrusca está inevitablemente sesgada por el valor de la muestra tan bajo. En este sentido, cabe mencionar que la decisión de compararla a pesar de la clara diferencia se debió a la poca variabilidad de uvas en parrilla y al no mantener la comparación solo en Isabella. En Isabella, donde los tamaños de muestra son más adecuados se observa una leve ventaja en producir en espaldera en el alto Espavey. En el caso del Bajo Espavey, la ventaja se mantiene por la parrilla. Sin embargo, gran parte de esa ventaja se debe a la longevidad de las plantas en parrilla y a la amplia área de cobertura que se les permite al dejar una distancia considerable y minimizar la poda.

También, reconocemos la existencia de limitaciones por superar en futuras investigaciones. Por ejemplo, no mostramos los datos correspondientes a la altura de las zonas analizadas debido a la carencia de equipo como GPS durante la realización del estudio.

Por otra parte, debido a los condicionamientos en el material, desconocemos las características fisicoquímicas y bioquímicas de las distintas variedades plantadas, por lo que no es posible determinar la calidad sobre la uva en sí misma o el vino que se puede producir de ellas con base en 
variables de crecimiento, estas últimas solo pueden sugerir información sobre la buena salud y condiciones apropiadas de las plantas con mayor y menor producción. A pesar de las limitaciones actuales con que cuenta el viñedo, sería recomendable a futuro, censar la calidad de las uvas evaluando las distintas propiedades bioquímicas por cada cuartel, con el fin de obtener el mejor rendimiento posible para cada variedad.

De momento, se recomienda calcular el promedio de producción (medido en kilogramos o toneladas) por variedad en cada cuartel donde se encuentren, manteniendo una muestra de al menos 30 plantas (al azar) correspondiente a cada una. En las etapas de vendimia, esta práctica podría contribuir en la toma de decisiones, con el fin de mejorar la productividad y ganancia del viñedo. Se recomienda a la vez realizar investigaciones en la Viña El Espavey, tomando en cuenta otras variables y aprovechar el trabajo conjunto, fundamentado en los aportes de la comunidad local y la comunidad universitaria en los procesos de investigación-acción social, en aras de plantear propuestas y desarrollar capacidades locales que beneficien a la sociedad costarricense, especialmente aquellos sectores más vulnerables, de manera que se enriquezca no sólo el saber comunitario sino también el quehacer académico.

\section{Agradecimiento y financiación}

El proyecto de las uvas es una iniciativa que evoca el valor del trabajo colaborativo e interdisciplinario, así como el contacto de la academia con las comunidades. En nombre del proyecto Enlace: plan institucional UCR-MEP para la inclusión educativa (TC 722), se agradece a Fabio Badilla, Isabella y Santiago Badilla Fallas, Isaac Badilla Blanco, a la comunidad de Chirriaca de Acosta, a los funcionarios de la Vicerrectoría de Acción Social de la Universidad de Costa Rica y a las personas colaboradoras de la Comunidad Universitaria por mirar más allá de los viñedos. El proyecto Enlace es financiado por la Universidad de Costa Rica.

\section{Referencias}

Abbal, P., Sablayrolles, J. M., Matzner-Lober, E., y Carbonneau, A. (2019). A Model for Predicting Wine Quality in a Rhône Valley Vineyard. Agronomy Journal, 111(2), 545-554. https://doi.org/10.2134/agronj2018.04.0269 
Almanza-Merchán, P. J. (2011). Determinación del crecimiento y desarrollo del fruto de vid (VitisviniferaL.) bajo condiciones de clima frío tropical (Tesis Doctoral). Universidad Nacional de Colombia.

Battaglia, M., Cherry, M. L., Beadle, C. L., Sands, P. J., y Hingston, A. (1998). Prediction of leaf area index in eucalypt plantations: effects of water stress and temperature. Tree Physiology, 18(8-9), 521-528. https://doi.org/10.1093/treephys/18.8-9.521

Bonilla, I. (2015). Análisis y manejo de la variabilidad intraparcelaria del viñedo en relación con la calidad de la uva y del vino (Tesis Doctoral). Universidad de La Rioja, España.

Camargo, U. A. (2005). Grape management techniques in tropical climates. En XIV International GESCO Viticulture Congress (pp. 251-256). Geisenheim, Alemania.

Camargo, U. A., Pereira, G. E., y Guerra, C. C. (2010). Wine grape cultivars adaptation and selection for tropical regions. En II International Symposium on Tropical Wines 910 (pp. 121-129). Petrolina, Brazil.

Cahoon, G. A. (1985). Potassium nutrition of grapes. Potassium in agriculture, (potassiuminagri), 1105-1134.

Coombe, B. G. (1987). Distribution of solutes within the developing grape berry in relation to its morphology. American Journal of Enology and Viticulture, 38(2), 120-127.

Commins, T., Asavasanti, S., y Deloire, A. (2012). What is tropical wine and what defines it? Thailand as a case study. Asian Journal of Food and Agro-Industry, 5(02), 79-95. http://www.ajofai.info/

Consejo Universitario. (2015). Políticas de la Universidad de Costa Rica 2016-2020. Universidad de Costa Rica.

Consejo Universitario. (2020). Políticas Institucionales 2021-2025. Universidad de Costa Rica.

Demir, K. O. K. (2014). A review on grape growing in tropical regions. TürkTarımveDoğaBilimleri Dergisi,1(Özel Say1-1), 1236-1241.

Disegna, E., Coniberti, A., y Dellacassa, E. (2005). Medición de área foliar de la vid: una herramienta para producir vinos de calidad. Revista INIA, 4, 18-20.

Herrero, M. V., Jiménez, A. E., Rojas, J. C., y Dobles, A. (1993). Índices de similitud entre taxocenosis de tlebótomos (Diptera: Psychodidae) en un área endémica de leishmaniasis cutánea en Costa Rica. Revista de biología tropical, 761-767. https://revistas.ucr.ac.cr/index.php/rbt/article/view/24090 
FAO STAT, F. (2010). Recuperado de: $<$ http://faostat. fao. org/site/567/default. aspx\# ancor $>$.Acessadoemsetembro.

González-Sanjosé, M. L., y Díez, C. (1992). Compuestos fenólicos en el hollejo de uva tinta durante la maduración. Agrochimica, 36(1), 1.

GrimaltTrò, M. D. M. (2016). Seguimiento fenológico de la variedad de uva" Moscatel"(Vitisvinifera L.) en la Marina Alta (Trabajo Fin de Grado). Universidad Miguel Hernández de Elche, España.

Jogaiah, S., Oulkar, D. P., Vijapure, A. N., Maske, S. R., Sharma, A. K., y Somkuwar, R. G. (2017). Influence of canopy management practices on fruit composition of wine grape cultivars grown in semi-arid tropical region of India. African Journal of Agricultural Research, 8(26), 3462-3472.

Keller, M., Mills, L. J., Wample, R. L., y Spayd, S. E. (2004). Crop load management in Concord grapes using different pruning techniques. American Journal of Enology and Viticulture, 55(1), 35-50.

Kliewer, W. M., y Weaver, R. J. (1971). Effect of Crop Level and Leaf Area on Growth, Composition, and Coloration ofTokay'Grapes. American Journal of Enology and Viticulture, 22(3), 172-177.

Lland, P., Gago, P., Caillard, A. y Dry, P. (2009). A Taste of the World of Wine. Patriclland Wine Promotions, Adelaide, South Australia.

López-Guzmán, T., Rodríguez García, J., \& Vieira Rodríguez, Áurea. (2013). Revisión de la literatura científica sobre enoturismo en España. Cuadernos De Turismo, (32), 171-188. Recuperado a partir de https://revistas.um.es/turismo/article/view/177511

Loría-Quirós, C. L. (2005). El injerto: alternativa de propagación vegetativa en el cultivo de la uva (Vitis vinifera) en Costa Rica. Revista de Agricultura Tropical, 35, 101-106.

Martínez de Toda Fernández, F. (1991). Biología de la vid: Fundamentos biológicos de la viticultura. España: Mundi Prensa Libros.

Pardo, J. A. (2016). Seguimiento Fenológico Del Cultivo de Uva (VitisSp) En Fusagasugá Cundinamarca (Tesis Doctoral). Universidad de Cundinamarca, Colombia.

Pinelo, M., Arnous, A., y Meyer, A. S. (2006). Upgrading of grape skins: Significance of plant cellwall structural components and extraction techniques for phenol release. Trends in FoodScience\& Technology, 17(11), 579-590. https://doi.org/10.1016/j.tifs.2006.05.003 
Possingham, J. V. (2006). Developments in the production of table grapes, wine and raisins in tropical regions of the world. En International Symposium on Grape Production and Processing 785 (pp. 45-50).

Reynier, A. (1989). Manual de viticultura. (4 ${ }^{\mathrm{a}}$ ed.). Madrid: Mundi-Prensa.

Ribéreau-Gayon, J., y Peynaud, E. (1971). Sciences et techniques de la vigne. Paris: Dunod.

Ribéreau-Gayon, J., Peynaud, E., Ribéreau-Gayon, P., y Sudraud, P. (1976). Sciences et techniques du vin. Paris: Bordas.

Sánchez, J. C., González, F. L., y Tena, A. M. (1999). Cultivo de la vid en espaldera. Cuadernos de divulgación. Consejería de Agricultura, Pesca y Alimentación. Gobierno de Canarias. España.

Tuanama, P., y Smith, H. (2019). Cuantificación de antocianinas por el método de pH diferencial del fruto de la uva Isabella (Vitis labrusca) procedente del distrito de San Antonio de Cumbaza. (Tesis). Universidad Nacional de San Maartin.

Uriarte, D., Mancha, L. A., Moreno, D., Gamero, E., Talaverano, M. I., Valdés, M. E., y Prieto, M. H. (2014). Efecto de la elevación de la altura de vegetación en un viñedo en espaldera del cv. Cigüente en Extremadura. I Jornadas del Grupo de Viticultura y Enología de la SECH Logroño, 19, 70-218. 